\title{
Exploitation of Heterosis in Single Cross Hybrids of Quality Protein Maize (Zea maize L.) for Yield and Quality Traits
}

\author{
Prashant Bisen*, Amit Dadheech, Namrata, Omprakash Nagar and Ram Kunwar Meena
}

Dept. of Plant Breeding and Genetics, Rajasthan College of Agriculture, Maharana Pratap University of Agriculture and Technology, Udaipur (313 001), India

\section{Corresponding Author}

Prashant Bisen

e-mail: prashant.bgt@gmail.com

\author{
Article History \\ Manuscript No. AR1748 \\ Received in $12^{\text {th }}$ Dec, 2016 \\ Received in revised form $28^{\text {th }}$ Jan, 2017 \\ Accepted in final form $6^{\text {th }}$ Feb, 2017
}

\begin{abstract}
Forty-five single-cross hybrids developed from ten inbred lines of quality protein maize through diallel mating design along with four checks viz., Pratap QPM Hybrid-1, Vivek QPM-9, HQPM-1 and HQPM-5 were evaluated in randomized block design with three replications for seventeen traits during kharif-2014, to identify the heterotic superiority of the New cross combinations over the parents and best check. Out of 45 crosses, 42 crosses over mid parent, 37 crosses over better parent and 6 crosses over standard check (HQPM-5) significantly out yielded for grain yield plant ${ }^{-1}$. Hybrid $\mathrm{P}_{6} \times \mathrm{P}_{8}$ showed maximum per se performance for grain yield plant ${ }^{-1}$, stover yield plant ${ }^{-1}$, ear length, ear girth and tryptophan content also showed good per se performance for oil content (6.13\%), starch content (69.83\%), protein content $(10.52 \%)$ and for lysine content $(4.19 \%)$ with maximum positive significant economic heterosis (19.63\%) for grain yield plant ${ }^{-1}$, over the best check HQPM-5. Hybrid $\mathrm{P}_{5} \times \mathrm{P}_{8}$ showed highest per se performance along with maximum positive significant economic heterosis for lysine content and protein content over the best check Vivek QPM-9 and HQPM-5, respectively. Another hybrid $P_{3} \times P_{5}$ and $P_{5} \times P_{7}$ exhibited highest per se performance for oil content and starch content, respectively, along with maximum positive significant economic heterosis over the best check HQPM-5 and HQPM-1, respectively.
\end{abstract}

Keywords: Heterosis, heterobeltiosis, QPM, grain yield, lysine, tryptophan

\section{Introduction}

Maize (Zea mays L.) occupies a prestigious place in the world agriculture. It is a miracle crop in view of its widespread usage as food and nonfood items (Lone et al., 2016). It is also considered as the third important cereal crop in the world after rice and wheat (Devi et al., 2016). Globally, maize has gained tremendous importance as a source of basic raw material for a no. of industries due to rising demand from diversified sectors like human food, animal feed and also serves. Maize grains contain about $9.9 \%$ protein, $4 \%$ oil, $70 \%$ starch and $2.7 \%$ crude fiber. Maize oil has high calorific value and is highly suitable especially for heart patients. Maize contains a high percentage of unsaturated fatty acids like oleic acid and linoleic acid and has a very low content of cholesterol. Maize has low protein content with low nutritional quality due to presence of limited amount of essential amino acids, such as lysine, tryptophan and methionine (Bantte and Prasanna, 2003; Huang et al., 2006; Mbuya et al., 2011) and an excess of leucine and isoleucine, leading to a poor growth in children and pellagra in adults.

Quality protein maize (QPM) is bio fortified maize with increased lysine and tryptophan levels. QPM contains higher amount of lysine and tryptophan in the endosperm ensuring higher biological value and availability of protein to human and animal so it can help to get rid of human malnourishment (Hussain et al., 2015). The biological value of common maize is $45 \%$ whereas that of QPM is about $80 \%$ (Rajendran et al., 2014). Substituting normal maize with high lysine maize on an equal weight basis can maintain proper amino acid balance (Wilson, 1991).

Maize is a highly cross pollinated crop and the scope for the exploitation of hybrid vigour depend on the direction and magnitude of heterosis (Reddy et al., 2015). Hybrids are preferred over varieties in maize for their yield potential. The breeding method to be adopted for maize improvement depends on the nature of the gene action involved in the expression of quantitative traits of economic importance, and its strength depends on the genetic variability in the base populations and development of superior inbreds (Rajendran et al., 2014). Especially, in heterosis breeding the choice of good combiners play a vital role (Singh et al., 2012). The breeding strategy for exploitation of heterosis in maize (Zea 
mays L.) through the cultivation of single cross hybrids is primarily dependent on the development and identification of high per se performing diverse, vigorous and productive inbred lines with good seed quality.

The grain yield is the primary trait targeted for improvement of maize productivity through exploitation of heterosis (Ulaganathan et al., 2015). To overcome the problems associated with multi-parent hybrids, a shift was made from multi-parent to two-parent hybrids. One of the ways to break the productivity barrier is to develop and popularize high yielding single cross hybrids (Kanagarasu et al., 2010; Farhan et al., 2012). In the view of above facts and in order to develop and identify productive, nutritionally superior and industrially important single cross hybrids, the present investigation for yield and quality traits was undertaken.

\section{Materials and Methods}

\subsection{Experimental site and design}

The experimental material consisted of ten diverse inbred lines (Table 1) of quality protein maize were crossed in all possible combinations using diallel mating design (excluding reciprocals) to obtain 45 single cross hybrids, during rabi season (March) of 2014 under irrigated, normal soil condition at the Instructional farm, Rajasthan College of Agriculture, Maharana Pratap University of Agriculture and Technology, Udaipur, India. These 45 hybrids, $10 \%$ along with four standard checks were evaluated in randomized block design with three replications, in a single row plot of $4 \mathrm{~m}$ length, maintaining crop geometry of $60 \times 25 \mathrm{~cm}^{2}$ in kharif-2014. The Recommended package of practices of zone IVA of Rajasthan were adopted to raise a healthy crop.

\subsection{Recording of data}

The data were recorded from five randomly selected competitive plants on seventeen distinct morphological and quality characters, except days to $50 \%$ tasseling, days to $50 \%$ silking and days to $75 \%$ brown husk, where it was observed on complete plot basis. The data on plant height, ear height, ear length, ear girth, no. of grain rows ear ${ }^{-1}$, 100-grain weight, grain yield plant ${ }^{-1}$, stover yield plant ${ }^{-1}$, Harvest index, grain oil content, starch content, protein content, tryptophan content and lysine content were recorded for statistical analysis.

\subsection{Biochemical analysis}

The total grain oil content was determined by Soxhlet Method (A.O.A.C., 1965), starch content by Anthrone reagent method (Morris, 1948), protein content by Micro-Kjeldahl's Method (Kalyana Babu et al., 2009).), tryptophan content by Papain Hydrolysis Method (Huang et al., 2006) and lysine content by using Colorimetric Method (Kalyana Babu et al., 2009).

\subsection{Statistical analysis}

The mean value of the recorded data was subjected to analysis of variance (ANOVA) using the statistical analysis procedures of Panse and Sukhatme, 1985. Relative heterosis/mid parent
Table 1: List of parental inbred lines and checks

\begin{tabular}{|c|c|c|c|}
\hline $\begin{array}{l}\text { Sl. } \\
\text { No. }\end{array}$ & $\begin{array}{l}\text { Inbred line } \\
\text { symbol/code }\end{array}$ & Pedigree & Source \\
\hline \multicolumn{4}{|c|}{ Details of parents } \\
\hline 1. & EIQ-105 $\left(P_{1}\right)$ & $\begin{array}{c}\text { CATCEYQ-72- } \\
5-4-2-2\end{array}$ & $\begin{array}{c}\text { AICRP on maize, } \\
\text { Udaipur }\end{array}$ \\
\hline 2. & ElQ-106 $\left(\mathrm{P}_{2}\right)$ & $\begin{array}{c}\text { CATCEYQ-72- } \\
2-1-1\end{array}$ & $\begin{array}{c}\text { AICRP on maize, } \\
\text { Udaipur }\end{array}$ \\
\hline 3. & EIQ-107 $\left(P_{3}\right)$ & $\begin{array}{c}\text { CATCEYQ-72- } \\
9-1-2-2\end{array}$ & $\begin{array}{c}\text { AICRP on maize } \\
\text { Udaipur }\end{array}$ \\
\hline 4. & EIQ-108 $\left(\mathrm{P}_{4}\right)$ & $\begin{array}{c}\text { CATCEYQ-72- } \\
10-3-4-2-1\end{array}$ & $\begin{array}{c}\text { AICRP on maize, } \\
\text { Udaipur }\end{array}$ \\
\hline 5. & EIQ-109 $\left(P_{5}\right)$ & $\begin{array}{c}\text { CATCEYQ-72- } \\
11-7-1-1-2\end{array}$ & $\begin{array}{l}\text { AICRP on maize, } \\
\text { Udaipur }\end{array}$ \\
\hline 6. & EIQ-110 $\left(P_{6}\right)$ & $\begin{array}{c}\text { CATCEYQ-72- } \\
9-3-6-1\end{array}$ & $\begin{array}{c}\text { AICRP on maize } \\
\text { Udaipur }\end{array}$ \\
\hline 7. & ElQ-111 $\left(\mathrm{P}_{7}\right)$ & $\begin{array}{c}\text { CATCEYQ-72- } \\
13-1-1-4\end{array}$ & $\begin{array}{c}\text { AICRP on maize } \\
\text { Udaipur }\end{array}$ \\
\hline 8. & EIQ-112 $\left(P_{8}\right)$ & $\begin{array}{l}\text { CATCEYQ-72- } \\
8-2-3-2-2\end{array}$ & $\begin{array}{l}\text { AICRP on maize, } \\
\text { Udaipur }\end{array}$ \\
\hline 9. & EIQ-113 $\left(P_{9}\right)$ & $\begin{array}{c}\text { CATCEYQ-72- } \\
5-2-3-1\end{array}$ & $\begin{array}{c}\text { AICRP on maize } \\
\text { Udaipur }\end{array}$ \\
\hline 10. & EIQ-114 $\left(P_{10}\right)$ & $\begin{array}{c}\text { CATCEYQ-72 } \\
-3\end{array}$ & $\begin{array}{c}\text { AICRP on maize } \\
\text { Udaipur }\end{array}$ \\
\hline
\end{tabular}

Details of checks

1. Pratap QPM hybrid-1 (Check-1)

AICRP on maize,
Udaipur
VPKAS. Almora
CCSHAU, Karnal
CCSHAU, Karnal

2. Vivek QPM- 9 (Check-2)

3. HQPM-1 (Check-3)

CCSHAU, Karnal

Where, AICRP: All India Coordinated Research Project;
VPKAS: Vivekanand Parvatiya Krishi Anusandhan Shala; CCSHAU: Choudhary Charan Singh Haryana Agricutural University

heterosis was calculated as per procedure suggested by Shull (1908). Heterobeltiosis/better parent heterosis was calculated as per procedure suggested by Fonesca and Patterson (1968). And Economic heterosis/standard heterosis were calculated as per procedure suggested by Briggle (1963).

\section{Results and Discussion}

The analysis of variance for experimental design (Table 2) revealed that the mean squares due to genotypes, parents, crosses and parents $\mathrm{v} / \mathrm{s}$ crosses were significant for all the traits. Significant mean squares due to parents and crosses suggested that the parental lines selected were diverse and with a different genetic background. Similarly, significant mean squares due to parents vs. crosses indicated presence 


\begin{tabular}{|c|c|c|c|c|c|c|c|c|c|c|}
\hline \multirow[t]{2}{*}{ SV } & \multirow[t]{2}{*}{$d f$} & \multicolumn{9}{|c|}{ Mean squares } \\
\hline & & $\begin{array}{c}\mathrm{DT} \\
50 \% \mathrm{~T} \\
\end{array}$ & $\begin{array}{c}\text { DT } \\
50 \% \mathrm{~S}\end{array}$ & $\begin{array}{c}\text { DT } \\
75 \% \text { BH }\end{array}$ & $\mathrm{PH}$ & $\mathrm{EH}$ & EL & EG & N GRE & $\begin{array}{l}100 \\
\text { GW } \\
\end{array}$ \\
\hline Replication & 2 & $13.82^{* *}$ & $6.56^{*}$ & $3.33^{*}$ & $177.19^{*}$ & $290.04^{* *}$ & 1.39 & 1.17 & 0.17 & 1.57 \\
\hline Genotype & 54 & $25.46^{* *}$ & $32.49^{* *}$ & $36.06^{* *}$ & $1211.56^{*}$ & $275.03^{* *}$ & $11.6^{* *}$ & $4.17^{* *}$ & $2.70^{* *}$ & $27.58^{* *}$ \\
\hline Parent & 9 & $31.87^{* *}$ & $31.90^{* *}$ & $36.70^{* *}$ & $574.60^{* *}$ & $330.60^{* *}$ & $11.72^{* *}$ & $3.78^{* *}$ & $3.70^{* *}$ & $8.11^{* *}$ \\
\hline Crosses & 44 & $19.55^{* *}$ & $29.10^{* *}$ & $32.75^{* *}$ & $673.23^{* *}$ & $192.82^{* *}$ & $10.05^{* *}$ & $2.98^{* *}$ & $2.24^{* *}$ & $28.08^{* *}$ \\
\hline Parent vs. crosses & 1 & $227.50^{* *}$ & $186.88^{*}$ & $176.00^{* *}$ & $30630.8^{*}$ & $339201^{*}$ & ${ }^{* *} \quad 97.82^{* *}$ & $60.10^{* *}$ & $14.30^{* *}$ & $180.51^{* *}$ \\
\hline Error & 108 & 0.85 & 0.80 & 0.95 & 56.08 & 27.30 & 0.54 & 0.56 & 1.13 & 0.83 \\
\hline \multicolumn{11}{|l|}{ Table 2: Continue... } \\
\hline \multirow[t]{2}{*}{ SV } & \multirow[t]{2}{*}{ d } & \multicolumn{9}{|c|}{ Mean squares } \\
\hline & & & GYP & SYP & $\mathrm{HI}$ & OC & SC & PC & TC & LC \\
\hline Replication & & 2 & 5.81 & 54.89 & 1.34 & 0.0004 & $1.13^{*}$ & 0.004 & 1.8 & 0.001 \\
\hline Genotype & & 54 & $32.0^{* *}$ & $934.73^{* *}$ & $30.88^{* *}$ & $1.55^{* *}$ & $21.33^{* *}$ & $3.004^{* *}$ & $0.03^{* *}$ & $1.30^{* *}$ \\
\hline Parent & & 12 & $7.49^{* *}$ & $256.08^{* *}$ & $18.31^{* *}$ & $1.55^{*}$ & $4.02^{* *}$ & $2.09^{* *}$ & $0.01^{* *}$ & $0.18^{* *}$ \\
\hline Crosses & & 44 & $3.21^{* *}$ & $734.79^{* *}$ & $27.90^{* *}$ & $1.32^{* *}$ & $18.70^{* *}$ & $2.30^{* *}$ & $0.024^{* *}$ & $0.99^{* *}$ \\
\hline Parent vs. crosses & & 14 & $41.8^{* *}$ & $15840.4^{* *}$ & $274.80^{* *}$ & $11.80^{* *}$ & $293.05^{* *}$ & $42.39^{* *}$ & $0.47^{* *}$ & $25.22^{* *}$ \\
\hline Error & & 108 & 0.41 & 19.09 & 2.55 & 0.001 & 0.323 & 0.004 & 4.13 & 0.0003 \\
\hline
\end{tabular}

SV: Source of variance; DT50\% T: Days to 50\% tasseling; DT50\% S: Days to 50\% silking; DT75\% BH: Days to 75\% brown husk; PH: Plant height; EH: Ear height; EL: Ear length; EG: Ear girth; NGRE: No. of grain rows ear-1; $100 \mathrm{GW:} 100$ grain weight; GYP-1: Grain yield plant ${ }^{-1}$; SYP-1: Stover yield plant ${ }^{-1} ;$ HI: Harvest index; OC: Oil content; SC: Starch content; PC: Protein content; TC: Tryptophan content; LC: Lysine content; ${ }^{*},{ }^{* *}$ Significant at $(p=0.05)$ and $(p=0.01)$ level of significance respectively

of considerable amount of variability and overall heterosis for all the traits under study. These results were in confirmation with Avinashe et al. (2013); Sundarajan and Kumar (2011).

\subsection{Per se performance}

The per se performance was advocated by Genter and Alexander (1962) as one of the method useful in evaluating parents for heterosis breeding in maize. The mean values of seventeen characters studied are presented in (Table 3).

A perusal of mean values of hybrids revealed that grain yield plant $^{-1}$ ranged from $50.00\left(P_{1} \times P_{3}\right)$ to $107.67 \mathrm{~g}\left(P_{6} \times P_{8}\right)$. The hybrid $\mathrm{P}_{6} \times \mathrm{P}_{8}$ showed maximum mean values for ear length $\left(16.17 \mathrm{~cm}\right.$.), ear girth $\left(13.33 \mathrm{~cm}\right.$.), grain yield plant ${ }^{-1}(107.67 \mathrm{~g}$ plant $^{-1}$ ) and stover yield plant ${ }^{-1}\left(137.33\right.$ g plant $\left.^{-1}\right)$. Hybrid $\mathrm{P}_{7} \times \mathrm{P}_{10}$ exhibited maximum mean value for 100 -grain weight $(31.83$ g). Hybrids $\mathrm{P}_{2} \times \mathrm{P}_{10}, \mathrm{P}_{4} \times \mathrm{P}_{5}, \mathrm{P}_{5} \times \mathrm{P}_{8}$ and $\mathrm{P}_{7} \times \mathrm{P}_{10}$ exhibited maximum mean value for no. of grain rows ear ${ }^{-1}$ (14.00). Three hybrids $\mathrm{P}_{1} \times \mathrm{P}_{8}, \mathrm{P}_{3} \times \mathrm{P}_{5}$ and $\mathrm{P}_{6} \times \mathrm{P}_{8}$ showed maximum mean values for ear girth $\left(13.33 \mathrm{~cm}\right.$.). Hybrid $\mathrm{P}_{1} \times \mathrm{P}_{9}$ exhibited maximum mean value for harvest index (47.86\%).

A perusal of data on mean values of maturity traits revealed that hybrid $\mathrm{P}_{2} \times \mathrm{P}_{4}$ exhibited minimum mean values for days to $50 \%$ silking ( 49.67 days), so we conclude that this hybrid comes under early maturity group.

The data on quality traits viz., oil, starch, protein, tryptophan and lysine content revealed that the hybrid $P_{3} \times P_{5}$ exhibited maximum mean values for oil content (6.23\%). The maximum mean value for starch content (70.02\%) was depicted by hybrid $\mathrm{P}_{5} \times \mathrm{P}_{7}$ whereas; hybrid $\mathrm{P}_{5} \times \mathrm{P}_{8}$ exhibited maximum mean value for protein content (10.58\%) and lysine content (4.25). The maximum mean value for tryptophan content $(0.94 \%)$ was depicted by hybrid $\mathrm{P}_{6} \times \mathrm{P}_{8}$.

\subsection{Magnitude of heterosis and heterobeltiosis}

A perusal of estimates of economic heterosis for grain yield plant ${ }^{-1}$ revealed that six hybrids $\mathrm{P}_{6} \times \mathrm{P}_{8}(19.63 \%), \mathrm{P}_{5} \times \mathrm{P}_{8}$ (15.56\%), $P_{3} \times P_{5}(11.11 \%), P_{5} \times P_{7}(10.00 \%), P_{1} \times P_{8}(8.89 \%)$ and $\mathrm{P}_{2} \times \mathrm{P}_{8}(7.22 \%)$ depicted positive significant economic heterosis for grain yield plant ${ }^{-1}$ over the best check HQPM- 5 (Table 4). Hybrid $\mathrm{P}_{6} \times \mathrm{P}_{8}$ showed maximum economic heterosis (19.63\%) for grain yield plant ${ }^{-1}$.

A perusal of estimates of economic heterosis for ear length revealed that only four hybrids $P_{6} \times P_{8}(15.48 \%), P_{3} \times P_{5}(14.29 \%)$, $P_{5} \times P_{8}(13.10 \%)$ and $P_{2} \times P_{8}(11.90 \%)$ depicted positive significant economic heterosis for ear length over the best check HQPM1. Six hybrids $P_{6} \times P_{8}(17.65 \%), P_{3} \times P_{5}(17.65 \%), P_{1} \times P_{8}(17.65 \%)$, $\mathrm{P}_{5} \times \mathrm{P}_{8}(14.71 \%), \mathrm{P}_{2} \times \mathrm{P}_{5}(11.76 \%)$ and $\mathrm{P}_{2} \times \mathrm{P}_{8}(11.76 \%)$ exhibited significant economic heterosis for ear girth over the best check Vivek QPM- 9 and HQPM-5. Only two hybrids $\mathrm{P}_{7} \times \mathrm{P}_{10}(6.70 \%)$ and $\mathrm{P}_{6} \times \mathrm{P}_{8}(5.59 \%)$ exhibited significant economic heterosis for 


\begin{tabular}{|c|c|c|c|c|c|c|c|c|}
\hline $\begin{array}{l}\text { Sl. } \\
\text { No. }\end{array}$ & Genotype & $\begin{array}{l}\text { Days to } 50 \% \\
\text { silking } \\
\end{array}$ & $\begin{array}{l}\text { Ear length } \\
(\mathrm{cm})\end{array}$ & $\begin{array}{l}\text { Ear girth } \\
(\mathrm{cm})\end{array}$ & $\begin{array}{l}\text { No. of grain } \\
\text { rows ear }{ }^{-1}\end{array}$ & $\begin{array}{l}100 \text {-grain } \\
\text { weight }(\mathrm{g})\end{array}$ & $\begin{array}{c}\text { Grain yield } \\
\text { plant }^{-1}(\mathrm{~g})\end{array}$ & $\begin{array}{c}\text { Stover yield } \\
\text { plant }^{-1}(\mathrm{~g})\end{array}$ \\
\hline \multicolumn{9}{|c|}{ Parents } \\
\hline 1. & $P_{1}$ & 63.33 & 10.67 & 8.50 & 10.67 & 19.67 & 35.33 & 71.33 \\
\hline 2. & $P_{2}$ & 62.00 & 6.83 & 8.67 & 10.67 & 20.33 & 48.67 & 80.00 \\
\hline 3. & $\mathrm{P}_{3}$ & 59.67 & 11.50 & 9.67 & 12.67 & 18.33 & 34.67 & 72.67 \\
\hline 4. & $\mathrm{P}_{4}$ & 64.67 & 11.33 & 10.17 & 12.67 & 18.33 & 45.67 & 80.00 \\
\hline 5. & $P_{5}$ & 64.33 & 8.33 & 9.17 & 10.67 & 20.00 & 44.67 & 80.67 \\
\hline 6. & $\mathrm{P}_{6}$ & 61.33 & 11.67 & 8.33 & 12.00 & 23.17 & 50.17 & 73.33 \\
\hline 7. & $P_{7}$ & 60.67 & 13.33 & 11.17 & 12.67 & 22.83 & 49.33 & 80.00 \\
\hline 8. & $P_{8}$ & 55.33 & 10.67 & 11.33 & 14.00 & 20.00 & 52.33 & 100.33 \\
\hline 9. & $P_{9}$ & 63.00 & 10.67 & 10.50 & 12.00 & 21.17 & 36.33 & 65.67 \\
\hline 10. & $P_{10}$ & 67.33 & 8.00 & 8.67 & 11.33 & 19.50 & 45.00 & 80.33 \\
\hline \multicolumn{9}{|c|}{ Hybrids } \\
\hline 1. & $\mathrm{P}_{1} \times \mathrm{P}_{8}$ & 55.67 & 14.83 & 13.33 & 13.33 & 25.33 & 98.00 & 132.33 \\
\hline 2. & $\mathrm{P}_{3} \times \mathrm{P}_{5}$ & 57.33 & 16.00 & 13.33 & 14.00 & 26.83 & 100.00 & 136.00 \\
\hline 3. & $P_{5} \times P_{7}$ & 58.67 & 12.67 & 12.50 & 12.00 & 23.17 & 99.00 & 128.67 \\
\hline 4. & $P_{5} \times P_{8}$ & 56.67 & 15.83 & 13.00 & 14.00 & 22.67 & 104.00 & 135.00 \\
\hline 5. & $\mathrm{P}_{6} \times \mathrm{P}_{8}$ & 55.67 & 16.17 & 13.33 & 12.00 & 31.50 & 107.67 & 137.33 \\
\hline \multicolumn{9}{|c|}{ Checks } \\
\hline 1. & Pratap QPM- 1 & 55.33 & 10.67 & 10.00 & 12.00 & 27.00 & 77.00 & 111.67 \\
\hline 2. & Vivek QPM- 9 & 51.00 & 12.83 & 11.33 & 14.00 & 29.83 & 80.33 & 110.00 \\
\hline 3. & HQPM- 1 & 61.67 & 14.00 & 11.00 & 12.67 & 25.67 & 86.00 & 120.00 \\
\hline \multirow[t]{8}{*}{4.} & HQPM- 5 & 61.67 & 12.50 & 11.33 & 14.00 & 26.33 & 90.00 & 131.67 \\
\hline & PM & 62.17 & 10.30 & 9.62 & 11.93 & 20.33 & 44.22 & 78.43 \\
\hline & FM & 59.41 & 12.30 & 11.18 & 12.70 & 23.05 & 68.39 & 103.84 \\
\hline & $\mathrm{CM}$ & 57.42 & 12.50 & 10.92 & 13.17 & 27.21 & 83.33 & 118.33 \\
\hline & GM & 58.60 & 11.84 & 10.75 & 12.41 & 22.54 & 64.54 & 99.15 \\
\hline & SEm \pm & 0.51 & 0.42 & 0.44 & 0.62 & 0.51 & 1.93 & 2.74 \\
\hline & $\mathrm{CD}(p=0.05)$ & 1.43 & 1.18 & 1.24 & 1.73 & 1.44 & 5.40 & 7.69 \\
\hline & $\mathrm{CD}(p=0.01)$ & 1.89 & 1.56 & 1.64 & 2.29 & 1.90 & 7.15 & 10.16 \\
\hline \multicolumn{9}{|c|}{ Table 3: Continue... } \\
\hline $\begin{array}{l}\text { Sl. } \\
\text { No. }\end{array}$ & Genotype & $\begin{array}{c}\text { Harvest } \\
\text { index (\%) }\end{array}$ & $\begin{array}{c}\text { Grain oil } \\
\text { content (\%) }\end{array}$ & $\begin{array}{c}\text { Starch } \\
\text { content (\%) }\end{array}$ & $\begin{array}{l}\text { Grain pr } \\
\text { conten }\end{array}$ & $\begin{array}{l}\text { tein } \\
(\%)\end{array}$ & $\begin{array}{l}\text { ryptophan } \\
\text { ontent (\%) }\end{array}$ & $\begin{array}{c}\text { Lysine content } \\
(\%)\end{array}$ \\
\hline \multicolumn{9}{|c|}{ Parents } \\
\hline 1. & $P_{1}$ & 33.13 & 3.24 & 59.72 & 8.0 & & 0.47 & 1.78 \\
\hline 2. & $P_{2}$ & 37.82 & 3.29 & 61.15 & 7.1 & & 0.51 & 1.81 \\
\hline 3. & $P_{3}$ & 32.39 & 3.14 & 62.96 & 8.4 & & 0.60 & 1.81 \\
\hline 4. & $\mathrm{P}_{4}$ & 36.29 & 4.21 & 62.44 & 8.1 & & 0.53 & 2.20 \\
\hline 5. & $P_{5}$ & 35.61 & 3.02 & 60.29 & 7.9 & & 0.58 & 2.11 \\
\hline 6. & $P_{6}$ & 40.63 & 4.96 & 62.04 & 6.0 & & 0.56 & 1.61 \\
\hline 7. & $P_{7}$ & 38.36 & 5.01 & 60.70 & 7.0 & & 0.56 & 1.99 \\
\hline
\end{tabular}




\begin{tabular}{|c|c|c|c|c|c|c|c|}
\hline $\begin{array}{l}\text { Sl. } \\
\text { No. }\end{array}$ & Genotype & $\begin{array}{l}\text { Harvest } \\
\text { index (\%) }\end{array}$ & $\begin{array}{c}\text { Grain oil } \\
\text { content (\%) }\end{array}$ & $\begin{array}{c}\text { Starch } \\
\text { content (\%) }\end{array}$ & $\begin{array}{c}\text { Grain protein } \\
\text { content }(\%)\end{array}$ & $\begin{array}{l}\text { Tryptophan } \\
\text { content (\%) }\end{array}$ & $\begin{array}{c}\text { Lysine } \\
\text { content (\%) }\end{array}$ \\
\hline 8. & $\mathrm{P}_{8}$ & 34.28 & 3.85 & 62.29 & 7.24 & 0.41 & 1.46 \\
\hline 9. & $P_{9}$ & 35.61 & 3.77 & 61.08 & 6.12 & 0.50 & 2.12 \\
\hline 10. & $P_{10}$ & 35.88 & 3.59 & 63.13 & 8.00 & 0.53 & 1.61 \\
\hline \multicolumn{8}{|c|}{ Hybrids } \\
\hline 1. & $P_{1} \times P_{8}$ & 42.55 & 5.82 & 69.44 & 10.29 & 0.93 & 4.02 \\
\hline 2. & $\mathrm{P}_{3} \times \mathrm{P}_{5}$ & 42.38 & 6.23 & 66.31 & 10.34 & 0.84 & 3.26 \\
\hline 3. & $\mathrm{P}_{5} \times \mathrm{P}_{7}$ & 43.48 & 4.71 & 70.02 & 9.75 & 0.67 & 2.92 \\
\hline 4. & $P_{5} \times P_{8}$ & 43.52 & 5.53 & 69.13 & 10.58 & 0.92 & 4.25 \\
\hline 5. & $\mathrm{P}_{6} \times \mathrm{P}_{8}$ & 43.95 & 6.13 & 69.83 & 10.52 & 0.94 & 4.19 \\
\hline 1. & Pratap QPM- 1 & 40.86 & 4.68 & 64.84 & 9.49 & 0.70 & 3.23 \\
\hline 2. & Vivek QPM- 9 & 42.20 & 4.17 & 67.79 & 8.65 & 0.81 & 4.16 \\
\hline 3. & HQPM- 1 & 41.79 & 4.93 & 68.12 & 10.05 & 0.91 & 3.73 \\
\hline \multirow[t]{8}{*}{4.} & HQPM- 5 & 40.61 & 5.77 & 68.00 & 10.16 & 0.75 & 3.49 \\
\hline & PM & 36.00 & 3.81 & 61.58 & 7.41 & 0.53 & 1.85 \\
\hline & FM & 39.35 & 4.50 & 65.04 & 8.72 & 0.66 & 2.86 \\
\hline & $\mathrm{CM}$ & 41.36 & 4.89 & 67.19 & 9.59 & 0.79 & 3.65 \\
\hline & GM & 38.31 & 4.35 & 63.53 & 8.42 & 0.64 & 2.72 \\
\hline & SEm \pm & 0.94 & 0.02 & 0.33 & 0.04 & 0.00 & 0.01 \\
\hline & $\mathrm{CD}(p=0.05)$ & 2.64 & 0.06 & 0.93 & 0.10 & 0.01 & 0.03 \\
\hline & $\mathrm{CD}(p=0.01)$ & 3.49 & 0.07 & 1.24 & 0.13 & 0.01 & 0.04 \\
\hline
\end{tabular}

Table 4: Five best hybrids identified on the basis of per se performance and economic heterosis for grain yield plant ${ }^{-1}$ along with their economic heterosis for quality traits

\begin{tabular}{llcccccccc}
\hline Sl. No. & Hybrids & PPGYP $(\mathrm{g})$ & EHGY $(\%)$ & DS & EHG $(\%)$ & EHS (\%) & EH (\%) & EHT (\%) & EHL (\%) \\
\hline 1. & $\left(\mathrm{P}_{6} \times \mathrm{P}_{8}\right)$ & 107.67 & $19.63^{* *}$ & 55.67 & $6.36^{* *}$ & $2.51^{* *}$ & $3.58^{* *}$ & $3.66^{* *}$ & $0.80^{* *}$ \\
2. & $\left(\mathrm{P}_{5} \times \mathrm{P}_{8}\right)$ & 104.00 & $15.56^{* *}$ & 56.67 & - & $1.48^{* *}$ & $4.14^{* *}$ & 1.10 & $2.08^{* *}$ \\
3. & $\left(\mathrm{P}_{3} \times \mathrm{P}_{5}\right)$ & 100.00 & $11.11^{* *}$ & 57.33 & $8.09^{* *}$ & - & $1.81^{* *}$ & - & - \\
4. & $\left(\mathrm{P}_{5} \times \mathrm{P}_{7}\right)$ & 99.00 & $10.00^{* *}$ & 58.67 & - & $2.78^{* *}$ & - & - & - \\
5. & $\left(\mathrm{P}_{1} \times \mathrm{P}_{8}\right)$ & 98.00 & $8.89^{* *}$ & 55.67 & $0.98^{* *}$ & $1.93^{* *}$ & $1.31^{* *}$ & $2.26^{* *}$ & - \\
6. & HQPM- 5 & 90.00 & - & 61.67 & - & - & - & - & - \\
7. & HQPM- 1 & 86.00 & - & 61.67 & - & - & - & - & - \\
8. & Vivek QPM-9 & 80.33 & - & 51.00 & - & - & - & - & - \\
9. & Pratap QPM & 77.00 & - & 55.33 & - & - & - & - & - \\
& hybrid- 1 & & & & & & & & - \\
\hline
\end{tabular}

PPGYP: Per se performance for grain yield plant ${ }^{-1}$; EHGY: Economic heterosis (\%) for grain yield plant ${ }^{-1}$ over the best check HQPM-5; DS: Days to 50\% silking; EHG: Economic heterosis (\%) for grain oil content over best check HQPM-5; EHS: Economic heterosis (\%) for starch content over best check HQPM-5; EH: Economic heterosis (\%) for grain protein content HQPM-5; EHT: Economic heterosis (\%) for tryptophan content over best check HQPM-1; EHL: Economic heterosis (\%) for lysine content over best check Vivek QPM-9

100 grain weight over the best check Vivek QPM-9. Only one hybrid namely $\mathrm{P}_{1} \times \mathrm{P}_{9}$ exhibited positive significant economic heterosis for harvest index (13.40\%) over the best check Vivek
QPM-9. Kumar et al. (2013); Netravati et al. (2013); Khan et al. (2014); Verma et al. (2014) also reported economic heterosis in maize for yield and its contributing traits. 
A perusal of quality traits revealed that only three hybrids depicted positive significant economic heterosis for grain oil content. The hybrid $\mathrm{P}_{3} \times \mathrm{P}_{5}$ exhibited maximum positive significant economic heterosis (8.09\%) for grain oil content over the best check HQPM-5. Five hybrids depicted positive significant economic heterosis for starch content. The hybrid $\mathrm{P}_{5} \times \mathrm{P}_{7}$ exhibited maximum positive significant economic heterosis for starch content $(2.78 \%)$ over the best check HQPM-1. In case of protein content five hybrids exhibited positive significant economic heterosis for this trait. The maximum positive significant economic heterosis for this trait was exhibited by hybrid $\mathrm{P}_{5} \times \mathrm{P}_{8}(4.14 \%)$ over the best check HQPM-5. For tryptophan contant only two hybrids exhibited positive significant economic heterosis. The maximum positive significant economic heterosis for this trait was exhibited by hybrid $\mathrm{P}_{6} \times \mathrm{P}_{8}(3.66 \%)$ over the best check HQPM-1. In case of lysine content four hybrids exhibited positive significant economic heterosis. Out of them the maximum positive significant economic heterosis was exhibited by hybrid $\mathrm{P}_{5} \times \mathrm{P}_{8}$ (2.08\%) over the best check Vivek QPM-9 (Table 4). Singh et al. (2013), Chahar et al. (2014), Lahane et al. (2014) reported economic heterosis for quality traits in maize. Only one hybrid namely $\mathrm{P}_{2} \times \mathrm{P}_{4}(-2.46 \%)$ exhibited negative significant economic heterosis for days to $75 \%$ brown husk. Five best identified hybrids having highest estimates of significant positive economic heterosis for grain yield plant ${ }^{-1}$ were viz., $\mathrm{P}_{6} \times \mathrm{P}_{8}, \mathrm{P}_{5} \times \mathrm{P}_{8}, \mathrm{P}_{3} \times \mathrm{P}_{5}, \mathrm{P}_{5} \times \mathrm{P}_{7}, \mathrm{P}_{1} \times \mathrm{P}_{8}$ (Table 5). Of these hybrids, few hybrids also showed higher estimates of significant positive economic heterosis for oil content $\left(\mathrm{P}_{6} \times \mathrm{P}_{8}\right.$ and $\left.\mathrm{P}_{1} \times \mathrm{P}_{8}\right)$, starch content $\left(P_{5} \times P_{7}, P_{6} \times P_{8}\right.$ and $\left.P_{5} \times P_{8}\right)$, protein content $\left(P_{5} \times P_{8^{\prime}} P_{6} \times P_{8}\right.$,

Table 5: Extent of heterosis for best five hybrids for yield, yield contributing and quality traits

\begin{tabular}{|c|c|c|c|c|c|c|c|c|c|c|}
\hline \multirow[t]{2}{*}{ SI. No. } & \multirow[t]{2}{*}{ Crosses } & \multicolumn{3}{|c|}{ Ear length $(\mathrm{cm})$} & \multicolumn{3}{|c|}{ Ear girth $(\mathrm{cm})$} & \multicolumn{3}{|c|}{ No. of grain rows ear ${ }^{-1}$} \\
\hline & & $\mathrm{RH}$ & $\mathrm{HB}$ & $\mathrm{EH}$ & $\mathrm{RH}$ & $\mathrm{HB}$ & $\mathrm{EH}$ & $\mathrm{RH}$ & $\mathrm{HB}$ & $\mathrm{EH}$ \\
\hline 1. & $\mathrm{P}_{1} \times \mathrm{P}_{8}$ & $39.06^{* *}$ & $39.06^{* *}$ & 5.95 & $34.45^{* *}$ & $17.65^{* *}$ & $17.65^{* *}$ & 8.11 & - & - \\
\hline 2. & $P_{3} \times P_{5}$ & $61.34^{* *}$ & $39.13^{* *}$ & $14.29^{* *}$ & $41.59^{* *}$ & $37.93^{* *}$ & $17.65^{* *}$ & $20.00^{* *}$ & 10.53 & 0.00 \\
\hline 3. & $\mathrm{P}_{5} \times \mathrm{P}_{7}$ & $16.92^{* *}$ & - & - & $22.95^{* *}$ & $11.94^{*}$ & 10.29 & 2.86 & - & - \\
\hline 4. & $\mathrm{P}_{5} \times \mathrm{P}_{8}$ & $66.67^{* *}$ & $48.44^{* *}$ & $13.10^{* *}$ & $26.83^{* *}$ & $14.71^{* *}$ & $14.71^{* *}$ & $13.51^{*}$ & 0.00 & $0.0-0$ \\
\hline 5. & $\mathrm{P}_{6} \times \mathrm{P}_{8}$ & $44.78^{* *}$ & $38.57^{* *}$ & $15.48^{* *}$ & $35.59^{* *}$ & $17.65^{* *}$ & $17.65^{* *}$ & -7.69 & - & - \\
\hline
\end{tabular}

\begin{tabular}{|c|c|c|c|c|c|c|c|c|c|c|}
\hline \multirow[t]{2}{*}{ SI. No. } & \multirow[t]{2}{*}{ Crosses } & \multicolumn{3}{|c|}{ 100-Grain weight (g) } & \multicolumn{3}{|c|}{ Grain yield plant $^{-1}(\mathrm{~g})$} & \multicolumn{3}{|c|}{ Stover yield plant ${ }^{-1}(\mathrm{~g})$} \\
\hline & & $\mathrm{RH}$ & $\mathrm{HB}$ & $\mathrm{EH}$ & $\mathrm{RH}$ & $\mathrm{HB}$ & $\mathrm{EH}$ & $\mathrm{RH}$ & $\mathrm{HB}$ & $\mathrm{EH}$ \\
\hline 1. & $\mathrm{P}_{1} \times \mathrm{P}_{8}$ & $27.73^{* *}$ & $26.67^{* *}$ & - & $123.57^{* *}$ & $87.26^{* *}$ & $8.89^{* *}$ & $54.17^{* *}$ & $31.89^{* *}$ & 0.51 \\
\hline 2. & $P_{3} \times P_{5}$ & $40.00^{* *}$ & $34.17^{* *}$ & - & $152.10^{* *}$ & $123.88^{* *}$ & $11.11^{* *}$ & $77.39^{* *}$ & $68.60^{* *}$ & 3.29 \\
\hline 3. & $P_{5} \times P_{7}$ & $8.17^{* *}$ & 1.46 & - & $110.64^{* *}$ & $100.68^{* *}$ & $10.00^{* *}$ & $60.17^{* *}$ & $59.50^{* *}$ & - \\
\hline 4. & $\mathrm{P}_{5} \times \mathrm{P}_{8}$ & $13.33^{* *}$ & $13.33^{* *}$ & - & $114.43^{* *}$ & $98.73^{* *}$ & $15.56^{* *}$ & $49.17^{* *}$ & $34.55^{* *}$ & 2.53 \\
\hline 5. & $\mathrm{P}_{6} \times \mathrm{P}_{8}$ & $45.95^{* *}$ & $35.97^{* *}$ & $5.59^{*}$ & $110.08^{* *}$ & $105.73^{* *}$ & $19.63^{* *}$ & $58.16^{* *}$ & $36.88^{* *}$ & 4.30 \\
\hline
\end{tabular}

\begin{tabular}{|c|c|c|c|c|c|c|c|c|c|c|}
\hline \multirow[t]{2}{*}{ SI. No. } & \multirow[t]{2}{*}{ Crosses } & \multicolumn{3}{|c|}{ Harvest index (\%) } & \multicolumn{3}{|c|}{ Grain oil content (\%) } & \multicolumn{3}{|c|}{ Starch content (\%) } \\
\hline & & $\mathrm{RH}$ & $\mathrm{HB}$ & $\mathrm{EH}$ & $\mathrm{RH}$ & $\mathrm{HB}$ & $\mathrm{EH}$ & $\mathrm{RH}$ & $\mathrm{HB}$ & $\mathrm{EH}$ \\
\hline 1. & $\mathrm{P}_{1} \times \mathrm{P}_{8}$ & $26.23^{* *}$ & $24.12^{* *}$ & 0.82 & $64.11^{* *}$ & $51.12^{* *}$ & $0.98^{*}$ & $13.82^{* *}$ & $11.48^{* *}$ & $1.93^{* *}$ \\
\hline 2. & $P_{3} \times P_{5}$ & $24.65^{* *}$ & $19.01^{* *}$ & 0.43 & $102.49^{* *}$ & $98.51^{* *}$ & $8.09^{* *}$ & $7.60^{* *}$ & $5.32^{* *}$ & - \\
\hline 3. & $P_{5} \times P_{7}$ & $17.56^{* *}$ & $13.35^{* *}$ & 3.04 & $17.36^{* *}$ & - & - & $15.74^{* *}$ & $15.35^{* *}$ & $2.78^{* *}$ \\
\hline 4. & $P_{5} \times P_{8}$ & $24.53^{* *}$ & $22.20^{* *}$ & 3.13 & $61.09^{* *}$ & $43.60^{* *}$ & - & $12.79^{* *}$ & $10.99^{* *}$ & $1.48^{*}$ \\
\hline 5. & $\mathrm{P}_{6} \times \mathrm{P}_{8}$ & $17.35^{* *}$ & $8.18^{*}$ & 4.15 & $39.18^{* *}$ & $23.66^{* *}$ & $6.36^{* *}$ & $12.34^{* *}$ & $12.11^{* *}$ & $2.51^{* *}$ \\
\hline \multicolumn{11}{|c|}{ Table 5: Continue... } \\
\hline \multirow[t]{2}{*}{ SI. No. } & \multirow[t]{2}{*}{ Crosses } & \multicolumn{3}{|c|}{ Grain protein content (\%) } & \multicolumn{3}{|c|}{ Tryptophan content (\%) } & \multicolumn{3}{|c|}{ Lysine content (\%) } \\
\hline & & $\mathrm{RH}$ & $\mathrm{HB}$ & $\mathrm{EH}$ & $\mathrm{RH}$ & $\mathrm{HB}$ & EH & $\mathrm{RH}$ & $\mathrm{HB}$ & $\mathrm{EH}$ \\
\hline 1. & $\mathrm{P}_{1} \times \mathrm{P}_{8}$ & $35.01^{* *}$ & $28.62^{* *}$ & $1.31^{*}$ & $112.12^{* *}$ & $97.18^{* *}$ & $2.56^{* *}$ & $147.43^{* *}$ & $125.23^{* *}$ & - \\
\hline 2. & $\mathrm{P}_{3} \times \mathrm{P}_{5}$ & $26.28^{* *}$ & $22.32^{* *}$ & $1.81^{* *}$ & $41.41^{* *}$ & $38.67^{* *}$ & - & $66.16^{* *}$ & $54.59^{* *}$ & - \\
\hline 3. & $P_{5} \times P_{7}$ & $30.54^{* *}$ & $23.10^{* *}$ & - & $18.48^{* *}$ & $16.09^{* *}$ & - & $42.28^{* *}$ & $38.45^{* *}$ & - \\
\hline 4. & $\mathrm{P}_{5} \times \mathrm{P}_{8}$ & $39.47^{* *}$ & $33.49^{* *}$ & $4.14^{* *}$ & $86.49^{* *}$ & $58.62^{* *}$ & 1.10 & $137.91^{* *}$ & $101.58^{* *}$ & $2.08^{* *}$ \\
\hline 5. & $\mathrm{P}_{6} \times \mathrm{P}_{8}$ & $58.08^{* *}$ & $45.24^{* *}$ & $3.58^{* *}$ & $94.50^{* *}$ & $67.46^{* *}$ & $3.66^{* *}$ & $172.89^{* *}$ & $160.46^{* *}$ & $0.80^{*}$ \\
\hline
\end{tabular}


$\mathrm{P}_{3} \times \mathrm{P}_{5}$ and $\left.\mathrm{P}_{1} \times \mathrm{P}_{8}\right)$, tryptophan content $\left(\mathrm{P}_{6} \times \mathrm{P}_{8}\right.$ and $\left.\mathrm{P}_{1} \times \mathrm{P}_{8}\right)$, lysine content $\left(\mathrm{P}_{5} \times \mathrm{P}_{8}\right.$ and $\left.\mathrm{P}_{6} \times \mathrm{P}_{8}\right)$.

Relative heterosis (MP) and heterobeltiosis (BP) are important parameters as they provide information about the presence of dominance and over dominance type of gene actions in the expression of various traits. Heterobeltiosis for grain yield plant ${ }^{-1}$ was exhibited by 37 hybrids with maximum heterobeltiosis depicted by the hybrid $\mathrm{P}_{1} \times \mathrm{P}_{9}$. This hybrid also exhibited significant positive heterobeltiosis for no. of yield contributing traits viz., ear length (18.75\%), 100 grain weight (20.47\%), stover yield plant ${ }^{-1}(27.57 \%)$ and harvest index $(34.38 \%)$, whereas for yield and yield contributing traits, the no. of hybrids depicting positive significant heterobeltiosis ranged from 6 (no. of grain rows ear ${ }^{-1}$ ) to 37 (grain yield plant ${ }^{-1}$ ) and for quality traits it varied from 25 (grain oil content) -44 (lysine content).

The presence of heterobeltiosis indicated that over dominance played an important role in the expression of all these traits. However, its magnitude and number of hybrids which exhibited significant heterobeltiosis were variable. Heterosis over better parent for grain yield was also reported by Amiruzzaman et al. (2013), Netravati et al. (2013); Chahar et al., (2014). Amanullah et al. (2011); Silva et al. (2011), Khanorkar et al. (2012) reported heterosis over better parent for maturity traits.

The study of relative heterosis revealed number of hybrids exhibiting positive significant mid parent heterosis for yield and yield contributing traits ranged from 15 (no. of grain rows ear $\left.{ }^{-1}\right)-42$ (grain yield plant ${ }^{-1}$ ). In case of quality traits, the no. of hybrids exhibiting positive significant mid parent heterosis ranged from 32 (grain oil content)-45 (tryptophan content and lysine content). For maturity traits, no. of hybrids exhibiting negative significant mid parent heterosis ranged from 28 (days to $75 \%$ brown husk)-34 (days to $50 \%$ silking). Similar findings for mid parent heterosis for yield and its contributing traits were also obtained by Khanorkar et al. (2012), Jain and Bhardawaj (2014); Ofori et al. (2015). Amanullah et al. (2011); Amiruzzaman et al. (2013) reported heterosis over better parent for maturity type traits.

\section{Conclusion}

Hybrid $\mathrm{P}_{6} \times \mathrm{P}_{8}$ could be identified as the best performing hybrid as it not only exhibited maximum positive economic heterosis (19.63\%) along with highest per se performance (107.67 g plant $^{-1}$ ) for grain yield plant ${ }^{-1}$, also exhibited positive economic heterosis for many yield contributing traits viz., ear length, ear girth, 100 grain weight, stover yield plant ${ }^{-1}$, harvest index and for quality traits viz., grain oil content, starch content, grain protein content, tryptophan content and lysine content. Hence these hybrids appear to be very promising combination for actual exploitation and could be recommended for testing in multi-location trials.

\section{References}

A.O.A.C., 1965. Official methods for oil analysis for association of Official Agricultural Chemists. $10^{\text {th }}$ (Edn.). Washington, D.C.

Amanullah, S.J., Muhammad, M., Muhammad, A.K., 2011. Heterosis studies in diallel crosses of maize. Sarhad Journal of Agriculture 27(2), 207-211.

Amiruzzaman, M., Islam, M.A., Lutful, H., Monjurul, K., Rohman, M.M., 2013. Heterosis and combining ability in a diallel among elite inbred lines of maize (Zea mays L.). Emirates Journal of Food and Agriculture 25(2), 132-137.

Avinashe, H.A., Jaiwar, S.S., Girase, V.K., Rawool, S.A., Khanorkar, S.M., 2013. Assessment of heterosis and combining ability for biochemical components in crosses among high quality protein maize (Zea mays L.). Journal of Soils and Crops 23(1), 176-184.

Bantte, K., Prasanna, B., 2003. Simple sequence repeat polymorphism in QPM lines. Euphytica 129, 33-344.

Briggle, L.W., 1963. Heterosis in wheat- a review. Crop Science 3, 407-412.

Chahar, S., Vyas, M., Ranwah, B.R., Vadodariya, G., 2014. Heterosis and combining ability analysis for yield and its contributing traits in early maturing maize (Zea mays L.) genotypes. Trends in Biosciences 7(14), 1774-1777.

Devi, S., Parimala, K., Sravanthi K., 2016. Gene action and combining ability analysis for yield and its component traits in maize and its component traits in maize (Zea mays L.). The Bioscan, 11(2), 1043-1047.

Farhan, A., Irfan, Ahmed, S., Hidayatur, R., Mohammad, N., Durrishahwar, Muhammad Yasir Khan, Ihteram, U., Jianbing, Y., 2012. Heterosis for yield and agronomic attributes in diverse maize germplasm. Australian Journal of Crop Science. 6(3), 455-462.

Fonseca, S., Patterson, F.L., 1968. Hybrid vigour in a seven parent diallel cross in common winter wheat (Triticum aestivum L.). Crop Science 8, 85-88.

Genter, G.F., Alexander, M.N., 1962. Comparitive performance of S1 progenies and test crosses of corn. Crop Science 2, 416-419.

Griffing, B., 1956. Concept of general and specific combining ability in relation to diallel crossing systems. Australian Journal of Biological Sciences 9, 463-493.

Huang, S., Frizzi, A., Florida, C.A., Kruger, D.E., Luethy, M.H., 2006. High lysine and high tryptophan transgenic maize resulting from the reduction of both $19-$ and $22-k D$ $\alpha$-zeins. Plant molecular biology 61(3), 525-535.

Hussain, M., Kiani, T.T., Shah, K.N., Ghafoor, A., Rabbani, A., 2015. Gene action studies for protein quality traits in (Zea mays L.) under normal and drought conditions. Pakistan Journal of Botany 47(1), 57-61.

Jain, R., Bharadwaj, D.N., 2014. Heterosis and inbreeding depression for grain yield and yield contributing characters in quality protein maize. Agricultural communications 2(1), 8-16.

Kalyana Babu, B., Agrawal, P.K., Mahajan, V., Gupta, H.S., 2009). Molecular and biochemical characterization of 
short duration quality protein maize. Journal of plant biochemistry and biotechnology 18(1), 93-96.

Kanagarasu, S., Nallathambi, G., Ganesan, K.N., 2010. Combining ability analysis for yield and its component traits in maize (Zea mays L.). Electronic Journal of Plant Breeding 1(4), 915-920.

Khan, R., Dubey, R.B., Vadodariya, G.D., Patel, A.I., 2014. Heterosis and combining ability for quantitative and quality traits in maize (Zea mays L.). Trends in Biosciences 7(6), 422-424.

Khanorkar, S.M., Avinashe, H.A., Jaiwar, S.S., Girase, V.K., 2012. Heterosis in quality protein maize (Zea mays L.). Maize Journal 1(1), 30-34.

Kumar, B., Razdan, A.K., Gupta B.B., 2013. Heterosis and character association for grain yield and its component traits in single cross maize hybrids under mid hills of jammu and kashmir, India. Agricultural Science Digest 33(3), 198-202.

Lahane, G.R., Chauhan, R.M., Patel, J.M., 2014. Combining ability and heterosis studies for yield and quality traits in quality protein maize. Journal of AgriSearch 1(3), 135-138.

Lone, A.A., Khan, M.H., Dar, Z.A., Wani, S.H., 2016. Breeding strategies for improving growth and yield under waterlogging conditions in maize: a review. Maydica 61(1), 1-11.

Mbuya, K., Nkongolo, K.K., Kalonji-Mbuyi, A., 2011. Nutritional analysis of quality protein maize varieties selected for agronomic characteristics in a breeding program. International Journal of Plant Breeding and Genetics 5, 317-327.

Morris, D.L., 1948. Quantitative determination of carbohyderate with Derwood's anthrone reagent. Science 107, 254-255.

Netravati, S.G., Adiger, S., Malkannavar, L., Gangashetty, P., 2013. Heterosis breeding for maturity, yield and quality characters in maize (Zea mays L.). Molecular Plant Breeding 6(4), 44-49.

Ofori, A.P., Ofori, K., Obeng-Antwi, K., Tengan, K.M.L., Badu-
Apraku, B., 2015. Combining ability and heterosis estimate of extra-early quality protein maize (QPM) single cross hybrids. Journal of Plant Breeding and Crop Science 7(4), 87-93.

Panse, V.G., Sukhatme, P.V., 1985. Statistical Methods for Agriculture Workers. ICAR, New Delhi, 14-33.

Reddy, V., Jabeen, F., Sudarshan, M.R., 2015. Heterosis studies in diallel crosses of maize for yield and yield attributing traits in maize (Zea mays L.) over locations. International Journal of Agriculture, Environment \& Biotechnology 8(2), 271.

Shull, G.H., 1908. What is heterosis. Genetics 33, 439-446.

Silva, V.Q.R., Amaral, J.A.T., Goncalves, L.S.A., Freitas, J.S.P., Candido, L.S., Vittorazzi, C., Moterle, L.M., Vieira, R.A., Scapim, C.A., 2011. Heterotic parameterizations of crosses between tropical and temperate lines of popcorn. Acta Scientiarum. Agronomy 33(2), 243-249.

Singh, P.K., Singh, A.K., Shahi, J.P., Ranjan, R., 2012. Combining ability and heterosis in quality protein maize. The Bioscan 7(2), 337-340.

Singh, P.K., Singh, N., Singh, A.K., Shahi, J.P., Rao, M., 2013. Heterosis in relation to combining ability in quality protein maize (Zea mays L.). Biolife 1(2), 65-69.

Sundararajan, R., Kumar, P.S., 2011. Studies on heterosis in maize (Zea mays L.). Plant Archives 11, 55-57.

Ulaganathan, V., Vinoth, R., Baghyalakshmi, K., Suvarna Rani, C., Gurusamy, A., 2015. Standard heterosis for grain yield and other agronomic characters in maize (Zea mays L.) under normal and moisture stress conditions. The Bioscan 10(3), 1251-1253.

Verma, R., Kumar, S.S., Reddy, V.N., Sankar, A.S., 2014. Heterosis studies for grain yield and its component traits in single cross hybrids of maize (Zea mays L.). International Journal of Plant, Animal and Environmental Science 4(1), 304-306.

Wilson, C.M., 1991. Multiple zeins from maize endosperms characterized by reverse phase high performance liquid chromatography. Plant Physiology 95, 777-786. 